\title{
Microwave-assisted preparation and antimicrobial activity of $\boldsymbol{O}$-alkylamino benzofurancarboxylates
}

\author{
Kinga Ostrowska $\cdot$ Elżbieta Hejchman $\cdot$ Irena Wolska • \\ Hanna Kruszewska • Dorota Maciejewska
}

Received: 16 February 2013/Accepted: 17 July 2013/Published online: 16 August 2013

(c) The Author(s) 2013. This article is published with open access at Springerlink.com

\begin{abstract}
A series of derivatives of 2 and 3-benzofurancarboxylates were synthesized under microwave-assisted conditions. Their in-vitro antimicrobial properties were assessed. Inhibition by the compounds of the growth of antibiotic-susceptible standards and clinically isolated strains of Gram-positive and Gram-negative bacteria, yeasts, and a human fungal pathogen was moderate to significant. Methyl 5-bromo-7-[2-( $N, N$-diethylamino)ethoxy]-6-methoxy-2-benzofurancarboxylate hydrochloride was identified as the most active compound (MIC $3-12 \times 10^{-3} \mu \mathrm{mol} / \mathrm{cm}^{3}$ against Gram-positive bacteria; MIC $9.4 \times 10^{-2} \mu \mathrm{mol} / \mathrm{cm}^{3}$ against Candida and Aspergillus brasiliensis). The molecular and crystal structures of 2-( $N, N$-diethylamino)ethyl 6-acetyl-5-hydroxy-2-methyl-3benzofurancarboxylate were established by single-crystal $\mathrm{X}$-ray diffraction.
\end{abstract}

Keywords Heterocycles - Alkylation .

Phase-transfer catalysis - X-ray structure determination . Drug research

K. Ostrowska $(\bowtie) \cdot$ E. Hejchman · D. Maciejewska

Department of Organic Chemistry, Faculty of Pharmacy,

Medical University of Warsaw,

1 Banacha, 02097 Warsaw, Poland

e-mail: kostrowska@wum.edu.pl

I. Wolska

Department of Crystallography, Faculty of Chemistry,

Adam Mickiewicz University, Grunwaldzka 6,

60780 Poznan, Poland

H. Kruszewska

Department of Antibiotics and Microbiology,

National Medicines Institute, 30/34 Chełmska,

00725 Warsaw, Poland

\section{Introduction}

The benzofuran system, an important pharmacophore, is present in numerous compounds isolated from natural sources and in synthetic products. These heterocyclic compounds have a variety of pharmacological properties, and changes of their structure result in high diversity that has proved useful in the search for new therapeutic agents. It is widely known that numerous compounds containing the benzo[b]furan system, both synthetic and isolated from natural sources, have antimicrobial activity [1].

Eight flavaglines and six cyclopenta[b]benzofurans isolated from Aglaia odorata, Aglaia elaeagnoidea, and Aglaia edulis (Meliaceae) have been tested for antifungal properties against the three plant pathogens Pyricularia grisea, Fusarium auenaceum, and Alternaria citri. P. grisea, responsible for rice blast disease, was the fungus most susceptible to all the benzofurans, with rocaglaol the most active compound [2]. Thirteen compounds based on the benzofuran structure bearing aryl substituents at the C-3 position through a methanone linker have been synthesized and screened for antibacterial and antifungal activity against four bacteria: Escherichia coli, Staphylococcus aureus, Methicillin-resistant S. aureus, and Bacillus subtilis, and a fungus Candida albicans. Four hydrophobic benzofuran analogs were found to have favorable antibacterial activity better than that of control drugs [3].

It has been shown that esters and amides of 4-substituted 2-benzofurancarboxylic acids may act as inhibitors of fungal $N$-myristoyltransferase [4-8]. Mild to significant inhibition of the growth of an antibiotic-susceptible standard, clinically isolated strains of Gram-positive and Gramnegative bacteria, and human fungal pathogens was observed for a series of 2-substituted and three new diacetyl benzofurans. Different substitution of the benzofuran 
moiety and subsequent antimicrobial screening identified the C-3-acetyl functionality as a new structural alternative for optimum antimicrobial activity in the benzofuran class of compounds [9]. Substituted 3-methyl-2-benzofurancarbohydrazides had moderate activity against $S$. aureus and B. subtilis [10]. Similarly, 2-(1-benzofuran-2-yl-)-5propyl-4,5-diphenyl-4,5-dihydrofuran-3-carbonitrile had average antimicrobial activity against $S$. aureus, B. subtilis, Pseudomonas aeruginosa, Micrococcus luteus, E. coli, Salmonella enteritidis, and Listeria monocytogenes [11]. Methyl esters of 4-bromo-6-(dibromoacetyl)-5-hydroxy-2methyl-1-benzofuran-3-carboxylic acid (I), 6-(dibromoacetyl)-5-methoxy-2-methyl-1-benzofuran-3-carboxylic acid (II), and 4-chloro-6-(dichloroacetyl)-5-hydroxy-2-methyl1-benzofuran-3-carboxylic acid (III) had antimicrobial activity against Gram-positive bacteria and compounds I and III had antifungal activity against Candida albicans and $C$. parapsilosis [12].

Surprisingly, no recently synthesized chloro and bromo derivatives of methyl 5-methoxy-2-methyl-3-benzofurancarboxylate had any antimicrobial activity [13].

As we have reported elsewhere, aminoalkylation the $\mathrm{OH}$ group of 7-hydroxycoumarin derivatives resulted in products with better antibacterial activity than the starting compounds [14]. Encouraged by this, and in continuation of our research, we designed the synthesis of a series of benzofurancarboxylates bearing $O$-aminoethyl substituents and assayed their antimicrobial activity. In this study we report their microwave-assisted preparation and discuss the advantages of this technique compared with synthesis under conventional conditions, described elsewhere [15].
The X-ray structure of 2-(N,N-diethylamino)ethyl 6-acetyl5-hydroxy-2-methyl-3-benzofurancarboxylate (1c) is presented, with inter and intramolecular interactions in the solid state.

\section{Results and discussion}

Our strategy was based on preparation of a series of derivatives of 2 and 3-benzofurancarboxylic acids (Fig. 1). Acids 1-6 were prepared as described elsewhere [15] and converted to their ammonium salts to improve solubility in polar solvents. Acids 1-4 and 6 were esterified with methanol to protect the carboxyl group against $O$-alkylation.

As the first step of our research we obtained $O$-alkylamino derivatives of methyl benzofurancarboxylates $\mathbf{1 b}$ $\mathbf{4 b}$ and $\mathbf{6 b}$ by microwave-assisted $O$-alkylation of the appropriate esters (compounds 1a-4a, 6a, Scheme 1, routes i and ii, Fig. 2), using 2-chloroethyl- $N, N$-diethylamine hydrochloride as alkylating agent.

The syntheses were performed in acetone under phasetransfer conditions, using anhydrous potassium carbonate as a base and Aliquat 336 ( $N$-methyl- $N, N$-dioctyloctan-1ammonium chloride) as phase-transfer catalyst (PTC). Preparation of hydrochloride salts of the resulting bases was necessary to prevent decomposition and improve their solubility in polar solvents. These compounds were previously synthesized conventionally [15]. Microwave assistance resulted in reduced reaction time (from 16 to $20 \mathrm{~h}$ to $24 \mathrm{~min}$ ); however, we did not notice any meaningful increase in product yield.
Fig. 1 Structures of 2 and 3-benzofurancarboxylic acids<smiles>CC(=O)c1cc2oc(C)c(C(=O)O)c2cc1O</smiles>

1<smiles>CC(=O)c1c(O)ccc2c(C)c(C(=O)O)oc12</smiles>

2<smiles>COc1cc2c(C)c(C(=O)O)oc2c(C(C)=O)c1O</smiles>

3<smiles>COc1ccc(/C=C/C(=O)c2c(O)ccc3c(C)c(C(=O)O)oc23)cc1</smiles> 
Scheme 1

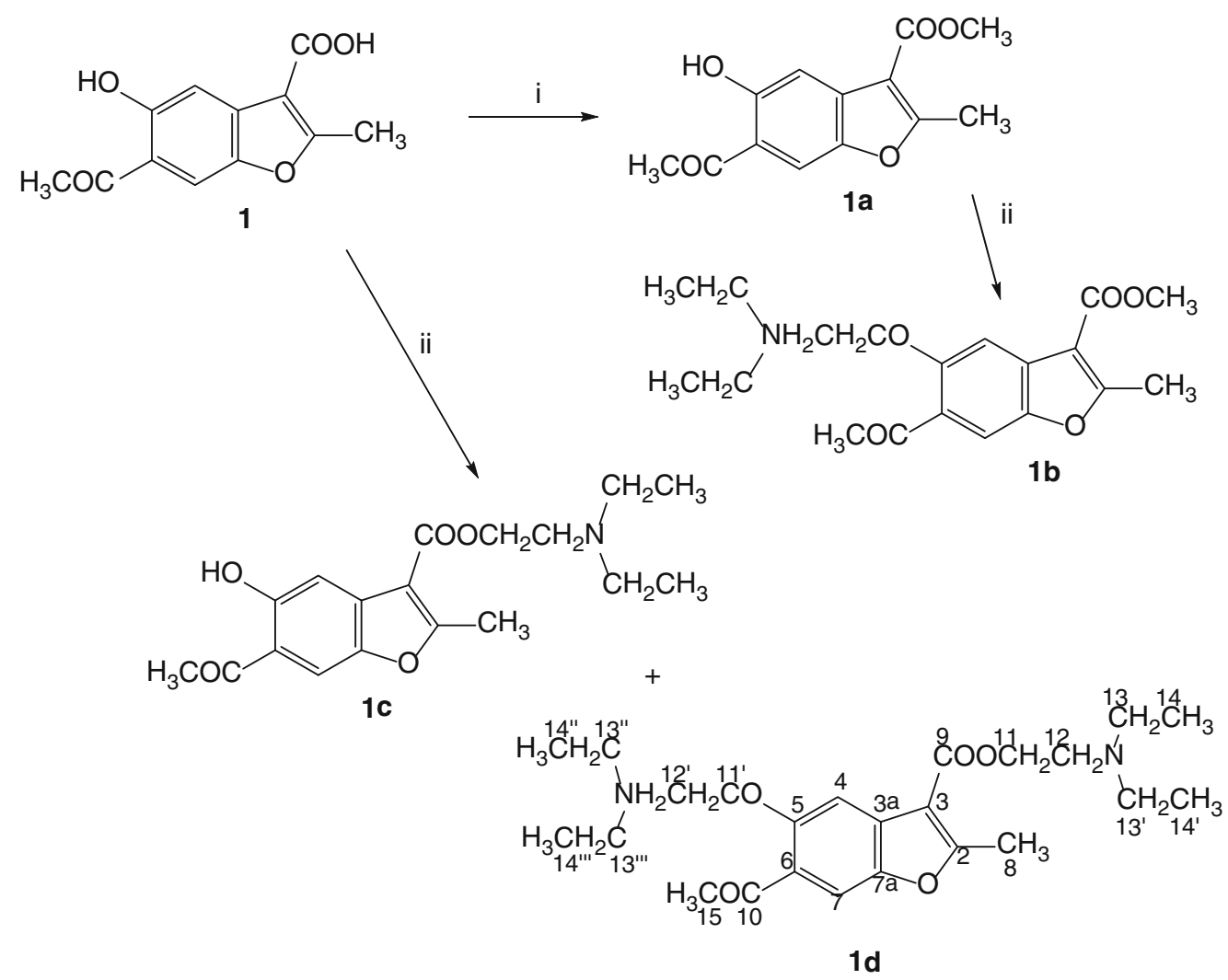

Benzofurancarboxylic acids 1-4, 6, and 7 reacted with 2-chloroethyl- $N, N$-diethylamine under similar conditions. Microwave-assisted alkylation of these compounds resulted in a mixture of two products. An example of this synthetic route (for compound 1) is presented in Scheme 1, route ii. Separation by column chromatography on silica gel yielded the product of esterification 1c and the product of $O$-alkylation and esterification 1d. The isolated compounds 1c, 1d, 3c, 4c, 6c, and 7c (Fig. 2) were converted to their hydrochloride salts. Spectroscopic data (IR, ${ }^{1} \mathrm{H}$ and ${ }^{13} \mathrm{C} \mathrm{NMR}$, and MS) confirmed the structures of all the products.

In this investigation eighteen derivatives of 2 and 3-benzofurancarboxylic acids were assayed for in-vitro antimicrobial activity. The ammonium salts of benzofurancarboxylic acids 1-7 (Fig. 1) were also tested. They did not inhibit the growth of any of the microorganisms $\left(\mathrm{MIC}>30 \mu \mathrm{mol} / \mathrm{cm}^{3}\right)$. Methyl esters 1a-7a of the acids [15] were not tested for antimicrobial activity.

Alkylation of hydroxyl groups in the molecules of methyl esters 1a-4a and $\mathbf{6 a}$ gave five 2-( $N, N$-diethylamino)ethoxy derivatives $\mathbf{1 b}-\mathbf{4 b}$ and $\mathbf{6 b}$ (Fig. 2; Scheme 1). All were evaluated microbiologically as hydrochloride salts. The in-vitro antimicrobial activity of compounds $\mathbf{1 b} \cdot \mathrm{HCl}-\mathbf{4 b} \cdot \mathrm{HCl}$ and $\mathbf{6 b} \cdot \mathrm{HCl}$ is summarized in Table 1.

The results show that the pattern of substitution of the benzofuran moiety is important to the activity. The most potent compound is $\mathbf{6 b} \cdot \mathrm{HCl}$; at concentrations in the range $3-12 \times 10^{-3} \mu \mathrm{mol} / \mathrm{cm}^{3}$ it inhibits growth of Gram-positive bacteria strains. Given its structure, we may speculate that the 2-(N,N-diethylamino)ethoxy function at $\mathrm{C}-7$, the bromine substituent at C-5, and the methoxy group at C-6 are responsible for the high activity. The isomeric compound $\mathbf{6 c} \cdot \mathrm{HCl}$ is, however, less active; exchanging the positions of the 2-( $N, N$-diethylamino)ethoxy and methoxy functions results in reduction of both antibacterial and antifungal activity.

It is worth noting that the derivative of the substituted 3-benzofurancarboxylic acid $\mathbf{1 b} \cdot \mathbf{H C l}$ is more active against Gram-positive bacteria strains than compounds $\mathbf{2 b} \cdot \mathbf{H C l}$, $\mathbf{3 b} \cdot \mathrm{HCl}$, and $\mathbf{4 b} \cdot \mathrm{HCl}$, obtained from the substituted 2-benzofurancarboxylic acids. Introducing the lipophilic methoxy group at the $\mathrm{C}-5$ position resulted in increased antimicrobial activity (compound $\mathbf{3 b} \cdot \mathbf{H C l}$ is more active then $\mathbf{2 b} \cdot \mathrm{HCl})$. Similarly, the 7-(p-methoxycinnamoyl $)$ group increases the activity of $\mathbf{4 b} \cdot \mathrm{HCl}$ compared with $\mathbf{2 b} \cdot \mathrm{HCl}$ against Gram-positive bacteria (Table 1). The 
Fig. 2 Structures of the esters of benzofurancarboxylic acids

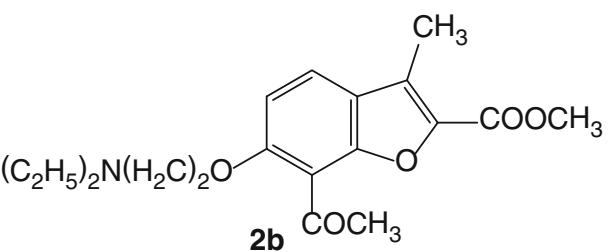

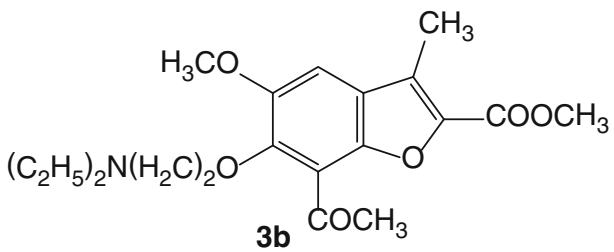<smiles>CCCCCOc1ccc2c(C)c(C(=O)OCCCC)oc2c1C(=O)/C=C/c1ccc(OC)cc1</smiles><smiles>CCN(CC)CCOc1c(OC)c(Br)cc2cc(C(=O)OC)oc12</smiles><smiles>[B]C1=C(C(=O)OCCCNCC)OC2C([14C](=O)[O-])=C(O)C(O[13CH3])=CC12</smiles>

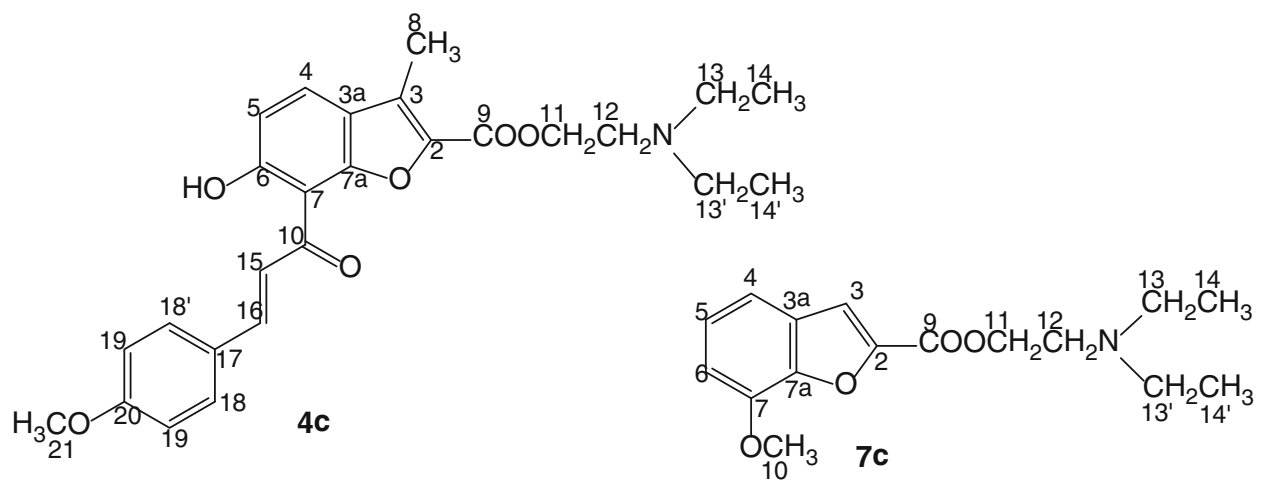<smiles>CCNCCCOC(=O)c1cc2cc(Br)c(OC)c(OC)c2o1</smiles>

2-(N,N-diethylamino)ethyl esters $\mathbf{1 c} \cdot \mathrm{HCl}, \mathbf{3 c} \cdot \mathrm{HCl}$, and $\mathbf{4 c} \cdot \mathrm{HCl}$, with unsubstituted phenolic groups, are more active against Gram-positive bacteria but less active against Gram-negative bacteria than $\mathbf{1 b} \cdot \mathrm{HCl}, \mathbf{3 b} \cdot \mathrm{HCl}$, and 4b. $\mathrm{HCl}$ (Table 2). It is worth noticing that compound $\mathbf{4 c} \cdot \mathrm{HCl}$ is the most active against yeast strains. Compound $\mathbf{7 c} \cdot \mathrm{HCl}$ was inactive in our assay.

\section{$X$-ray structure analysis}

The molecular and crystal structure of $\mathbf{1 c}$ in the solid state were analyzed by single-crystal X-ray diffraction. The molecular structure with the atomic numbering scheme is illustrated in Fig. 3 (the drawings were performed with Mercury software [16]). The results indicate that the compound crystallizes in the monoclinic space group $P 22_{1} / n$ with one molecule in the asymmetric unit. Selected bond lengths, bond angles, and torsion angles are listed in Table 3 . The benzofuran moiety is nearly planar with a maximum deviation of $0.020(1) \AA$ for $\mathrm{C} 3 \mathrm{a}$. The C8, C9, C10, O16, O17, and $\mathrm{O} 18$ atoms are almost coplanar with the two-ring framework (the appropriate torsion angles are given in Table 3). The orientation of the substituent at $\mathrm{C} 3$ relative to the benzofuran ring can be described by the torsion angle $\mathrm{C} 2-\mathrm{C} 3-\mathrm{C} 9-\mathrm{O} 19$ of $-0.2(3)^{\circ}$. For the $(N, N-$ diethylamino)ethyl fragment we observed structural disorder as a result of conformational freedom and from X-ray data we found alternative positions of the $\mathrm{C} 12$ and $\mathrm{C} 13$ atoms. Strong intramolecular hydrogen bonding is present between $\mathrm{O} 16$ and $\mathrm{O} 17$ atoms (Fig. 3; Table 4). The angle 
Table 1 Antimicrobial activity of hydrochlorides of methyl benzofurancarboxylate $O$-alkylamino derivatives (minimum inhibitory concentration, $\mu \mathrm{mol} \mathrm{cm}-3$ )

\begin{tabular}{|c|c|c|c|c|c|}
\hline & $\mathbf{1 b} \cdot \mathrm{HCl}$ & $\mathbf{2 b} \cdot \mathrm{HCl}$ & $\mathbf{3 b} \cdot \mathrm{HCl}$ & $\mathbf{4 b} \cdot \mathrm{HCl}$ & $6 \mathbf{b} \cdot \mathrm{HCl}$ \\
\hline Micrococcus luteus & 0.05 & 0.75 & 0.05 & 0.04 & 0.003 \\
\hline \multicolumn{6}{|l|}{ ATCC 9341} \\
\hline Bacillus cereus & 0.05 & 1.49 & 0.36 & 0.30 & 0.012 \\
\hline \multicolumn{6}{|l|}{ ATCC 11178} \\
\hline Bacillus subtilis & 0.05 & 1.49 & 0.18 & 0.04 & 0.012 \\
\hline \multicolumn{6}{|l|}{ ATCC 6633} \\
\hline Staphylococcus epidermidis & 0.05 & 1.49 & 0.18 & 0.04 & 0.012 \\
\hline \multicolumn{6}{|l|}{ ATCC 12228} \\
\hline Staphylococcus aureus & 0.10 & 3.11 & 0.18 & 0.15 & 0.012 \\
\hline \multicolumn{6}{|l|}{ ATCC 6538} \\
\hline Staphylococcus aureus & 0.05 & 3.11 & 0.18 & 0.15 & 0.012 \\
\hline \multicolumn{6}{|l|}{ ATCC $6538 \mathrm{P}$} \\
\hline Enterococcus hirae & 0.39 & 3.11 & 0.36 & 0.60 & 0.012 \\
\hline \multicolumn{6}{|l|}{ ATCC 10541} \\
\hline Escherichia coli & 6.51 & 12.44 & 6.04 & NA & 1.50 \\
\hline \multicolumn{6}{|l|}{ ATCC 8739} \\
\hline Pseudomonas aeruginosa & 13.02 & NA & NA & NA & 3.12 \\
\hline \multicolumn{6}{|l|}{ ATCC 15442} \\
\hline Candida albicans & 0.78 & 1.49 & 0.36 & 4.98 & 0.09 \\
\hline \multicolumn{6}{|l|}{ ATCC 10231} \\
\hline Candida albicans & 0.39 & 1.49 & 0.36 & 4.98 & 0.09 \\
\hline \multicolumn{6}{|l|}{ ATCC 2091} \\
\hline Candida parapsilosis & 0.39 & 1.49 & 0.72 & 0.2987 & 0.094 \\
\hline \multicolumn{6}{|l|}{ ATCC 22019} \\
\hline Saccharomyces cerevisiae & NT & NT & NT & NT & 0.187 \\
\hline \multicolumn{6}{|l|}{ ATCC 9763} \\
\hline Zygosacharomyces rouxi & 0.39 & NT & 0.36 & NT & 0.023 \\
\hline \multicolumn{6}{|l|}{ ATCC 28253} \\
\hline Aspergillus brasiliensis & 0.78 & 1.49 & 0.72 & 1.19 & 0.094 \\
\hline ATCC 16404 & & & & & \\
\hline
\end{tabular}

$N A$ not assayed $>0.3 \mu \mathrm{mol} / \mathrm{cm}^{3}, N T$ not tested

between the best planes of the benzofuran moiety and the $\mathrm{C} 5 / \mathrm{O} 17 / \mathrm{H} 17 \mathrm{~A} / \mathrm{O} 16 / \mathrm{C} 10 / \mathrm{C} 6 \mathrm{ring}$ is only $1.56(6)^{\circ}$. Moreover the weak $\mathrm{C} 4-\mathrm{H} 4 \mathrm{~A} \cdots \mathrm{O} 18$ and $\mathrm{C} 11-\mathrm{H} 11 \cdots \mathrm{O} 18$ interactions stabilize the conformation of the molecule.

The packing of the molecules viewed down the $a$ axis (Fig. 4) shows that the molecules are stacked in blocks with partly overlapping benzofuran systems and an interlayer spacing of ca. $3.5 \AA$. The molecules are linked by C7-H7A...O18, C11-H11A(D)‥O1 hydrogen bonds forming infinite chains along the $a$ axis. These chains interact via C13D-H13G‥O17, C15-H15C...C9, C8H8B $\cdots \mathrm{C} 10$ contacts and $\pi \cdots \pi$ stacking forces to create the blocks mentioned above. The bulky aminoethyl substituents are oriented outside these blocks and connect them via C13C-H13F...O16 hydrogen bonds. Geometric data for all intra and intermolecular interactions are given in Table 4.

\section{Experimental}

Reagents of the highest grade available were purchased from Aldrich and used without further purification. Solvents were used as received from commercial suppliers, and no further attempts were made to purify or dry them. Melting points were determined with an ElectroThermal 9001 digital melting point apparatus (ElectroThermal, Essex, UK). A Plazmatronika 1,000-W microwave oven equipped with a single mode cavity suitable for microscale synthesis and microwave choked outlet connected to an external condenser set to $30 \%$ power was used (http:// www.plazmatronika.com.pl). High-resolution mass spectra were recorded on a Quattro LCT (TOF). ${ }^{1} \mathrm{H}$ NMR, ${ }^{13} \mathrm{C}$ NMR, HSQC, and HMBC spectra in solution were recorded at $25{ }^{\circ} \mathrm{C}$ with Varian NMRS-300 or a Varian Unity 
Table 2 Antimicrobial activity of hydrochlorides of 2-(N,N-diethylamino)ethyl benzofurancarboxylates (minimum inhibitory concentration, $\mu \mathrm{mol} \mathrm{cm} \mathrm{cm}^{-3}$ )

\begin{tabular}{|c|c|c|c|c|c|c|}
\hline & 1c $\cdot \mathrm{HCl}$ & $3 c \cdot \mathrm{HCl}$ & $4 \mathrm{c} \cdot \mathrm{HCl}$ & $\mathbf{6 c} \cdot \mathrm{HCl}$ & $\mathbf{1 d} \cdot \mathrm{HCl}$ & $7 \mathrm{c} \cdot \mathrm{HCl}$ \\
\hline $\begin{array}{l}\text { Micrococcus luteus } \\
\text { ATCC } 9341\end{array}$ & 0.01 & 0.01 & 0.01 & 0.09 & 0.04 & 15.28 \\
\hline $\begin{array}{l}\text { Bacillus cereus } \\
\text { ATCC } 11778\end{array}$ & 0.10 & 0.05 & 0.04 & 0.71 & 0.04 & 15.28 \\
\hline $\begin{array}{l}\text { Bacillus subtilis } \\
\text { ATCC } 6633\end{array}$ & 0.01 & 0.09 & 0.04 & 0.35 & 0.04 & 15.28 \\
\hline $\begin{array}{l}\text { Staphylococcus epidermidis } \\
\text { ATCC } 12228\end{array}$ & NA & 0.19 & 0.01 & 0.09 & 0.04 & 15.28 \\
\hline $\begin{array}{l}\text { Staphylococcus aureus } \\
\text { ATCC } 6538\end{array}$ & 1.62 & 0.19 & 0.01 & 0.09 & 0.04 & 15.28 \\
\hline $\begin{array}{l}\text { Staphylococcus aureus } \\
\text { ATCC } 6538 \mathrm{P}\end{array}$ & 0.41 & 0.09 & 0.04 & 0.18 & 0.07 & 15.28 \\
\hline $\begin{array}{l}\text { Enterococcus hirae } \\
\text { ATCC } 10541\end{array}$ & NA & 0.75 & 0.08 & NA & 0.30 & $>30.56$ \\
\hline $\begin{array}{l}\text { Escherichia coli } \\
\text { ATCC } 8739\end{array}$ & NA & NA & NA & NA & 0.59 & $>30.56$ \\
\hline $\begin{array}{l}\text { Pseudomonas aeruginosa } \\
\text { ATCC } 15442\end{array}$ & NA & NA & NA & NA & NA & 30.56 \\
\hline $\begin{array}{l}\text { Candida albicans } \\
\text { ATCC } 10231\end{array}$ & 1.62 & NA & 0.32 & 5.91 & 2.47 & $>30.56$ \\
\hline $\begin{array}{l}\text { Candida albicans } \\
\text { ATCC } 2091\end{array}$ & 0.41 & NA & 0.08 & NA & 4.96 & $>30.56$ \\
\hline $\begin{array}{l}\text { Candida parapsilosis } \\
\text { ATCC } 22019\end{array}$ & NA & NA & 0.15 & NA & 4.96 & $>30.56$ \\
\hline $\begin{array}{l}\text { Saccharomyces cerevisiae } \\
\text { ATCC } 9763\end{array}$ & 1.62 & 3.13 & 0.08 & 1.42 & 4.96 & 15.28 \\
\hline $\begin{array}{l}\text { Zygosacharomyces rouxi } \\
\text { ATCC } 28253\end{array}$ & NA & NA & 0.04 & 5.91 & 0.59 & $>30.56$ \\
\hline $\begin{array}{l}\text { Aspergillus brasiliensis } \\
\text { ATCC } 16404\end{array}$ & NA & NA & NA & NA & 4.96 & 15.28 \\
\hline
\end{tabular}

$N A$ not assayed $>0.3 \mu \mathrm{mol} / \mathrm{cm}^{3} ; N T$ not tested

Fig. 3 Schematic diagram of molecule 1c showing the labeling scheme and the disordered 2-(N,Ndiethylamino)ethyl substituent

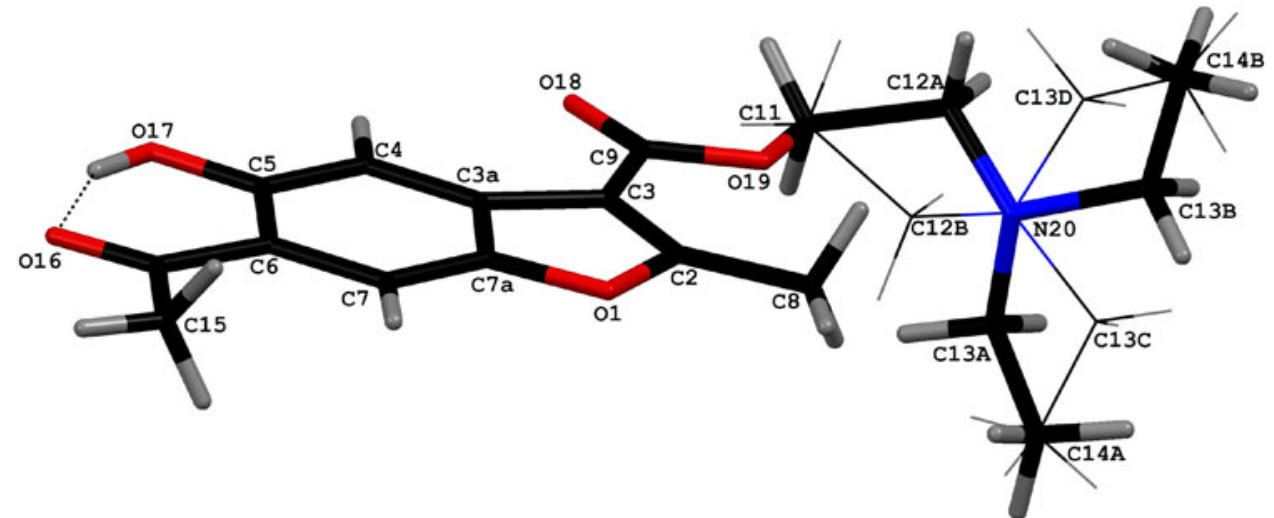

plus-500 spectrometers, and standard Varian software was used (Varian, Palo Alto, CA, USA). Calculated shielding constants were used as an aid to assignment of resonances of ${ }^{13} \mathrm{C}$ atoms. The CPHF-GIAO approach was used for computation of NMR shielding constants using Gaussian 09 software [17]. Chemical shifts $(\delta, \mathrm{ppm})$ were referenced 
to TMS. The notation used for detailed description of NMR resonances is given in Scheme 1 and Fig. 2. IR spectra were recorded on a Perkin Elmer FT IR Spectrum 2000 instrument. TLC was performed on silica gel $60 \mathrm{~F}_{254}$ sheets (Merck, Darmstadt, Germany), spots were visualized by UV at 254 and $365 \mathrm{~nm}$. Silica gel 60 was used for column chromatography. Preparation of compounds $\mathbf{1 b}-\mathbf{6 b}$ has been described elsewhere [15].

General procedure for microwave-assisted preparation of hydrochlorides of methyl [2-(N,N-diethylamino)ethoxy]-substituted benzofurancarboxylates

A mixture of the appropriate methyl benzofurancarboxylate (2 mmol), N,N-diethyl-2-chloroethylamine hydrochloride (6 mmol), anhydrous potassium carbonate $(23 \mathrm{mmol})$, and Aliquat $336(0.25 \mathrm{mmol})$ in $10 \mathrm{~cm}^{3}$ anhydrous acetone was placed in the microwave flask and heated under reflux in the

Table 3 Selected bond lengths/A and angles $/{ }^{\circ}$, and selected torsional angles $/{ }^{\circ}$ for $\mathbf{1 c}$

\begin{tabular}{ll}
\hline O1-C2 & $1.309(2)$ \\
O1-C7a & $1.384(2)$ \\
C5-O17 & $1.351(2)$ \\
C3a-C7a & $1.331(2)$ \\
C6-C10 & $1.433(3)$ \\
C2-C8 & $1.477(2)$ \\
C2-O1-C7a & $108.6(1)$ \\
C3-C9-O19 & $112.5(1)$ \\
C6-C10-O16 & $123.2(2)$ \\
C3a-C3-C2-C8 & $177.1(2)$ \\
C7-C6-C5-O17 & $179.0(2)$ \\
C2-C3-C9-O18 & $178.8(2)$ \\
O1-C2-C3-C9 & $-178.3(2)$ \\
C7-C6-C10-O16 & $-178.1(2)$ \\
C4-C5-C6-C10 & $179.6(2)$ \\
\hline
\end{tabular}

monomode microwave oven for $24 \mathrm{~min}$. The reaction was monitored by TLC. After completion of the reaction inorganic salts were removed by filtration. The solvent was evaporated. The residue was purified by column chromatography on silica gel, eluent: $\mathrm{CHCl}_{3}-\mathrm{MeOH}$ 50:1. The base was dissolved in methanol saturated with gaseous $\mathrm{HCl}$. The hydrochloride was precipitated by addition of diethyl ether. The crude product was crystallized from methanoldiethyl ether.

Methyl 6-acetyl-5-[2-(N,N-diethylamino)ethoxy]-2-methyl3-benzofurancarboxylate (1b, $\left.\mathrm{C}_{19} \mathrm{H}_{25} \mathrm{NO}_{5}\right)$

${ }^{1} \mathrm{H} \mathrm{NMR}\left(300 \mathrm{MHz}, \mathrm{CDCl}_{3}\right): \delta=1.11(\mathrm{t}, J=7.2 \mathrm{~Hz}, 6 \mathrm{H}$, H-14,14'), 2.69 (s, 3H, H-16), 2.70 (m, 4H, H-13,13'), 2.77 (s, 3H, H-8), 2.99 (t, J = 6.3 Hz, 2H, H-12), 3.96 (s, 3H, H-15), 4.25 (t, J = 6.3 Hz, 2H, H-11), 7.49 (s, 1H, H-4), $7.82(\mathrm{~s}, 1 \mathrm{H}, \mathrm{H}-7) \mathrm{ppm} ;{ }^{13} \mathrm{C} \mathrm{NMR}\left(125 \mathrm{MHz}, \mathrm{CDCl}_{3}\right)$ : $\delta=11.66\left(\mathrm{C}-14,14^{\prime}\right), 15.10(\mathrm{C}-8), 32.16$ (C-16), 47.92 (C-13), 51.81 (C-15), 52.02 (C-12), 67.45 (C-11), 104.70 (C4), 109.25 (C-3), 112.51 (C-7), 125.88 (C-6), 131.05 (C-3a), 148.20 (C-7a), 155.68 (C-5), 164.62 (C-9), 167.40 (C-2), 199.34 (C-10) ppm.

1b. $\mathrm{HCl}\left(\mathrm{C}_{19} \mathrm{H}_{26} \mathrm{ClNO}_{5}\right)$

${ }^{1} \mathrm{H}$ NMR $\left(300 \mathrm{MHz}, \mathrm{CDCl}_{3}\right): \delta=1.48(\mathrm{t}, J=6.9 \mathrm{~Hz}, 6 \mathrm{H}$, H-14,14' ), 2.62 (s, 3H, H-16), 2.71 (m, 4H, H-13,13'), 2.79 (br.s, 3H, H-8), 3.21 (m, 4H, H-13, 13'), 3.55 (m, 2H, H-12), 3.98 (s, 3H, H-15), 4.71 (br.s, 2H, H-11), 7.55 (s, 1H, H-4), 7.74 (s, 1H, H-7), 12.47 (br.s, 1H, NH) ppm.

Methyl 7-acetyl-6-[2-(N,N-diethylamino)ethoxy]-3-methyl2-benzofurancarboxylate hydrochloride

(2b. $\mathrm{HCl}, \mathrm{C}_{19} \mathrm{H}_{26} \mathrm{ClNO}_{5} x \mathrm{H}_{2} \mathrm{O}$ ), methyl 7-acetyl-6-[2-(N,N-diethylamino)ethoxy]-5methoxy-3-methyl-2-benzofurancarboxylate hydrochloride $\left(\mathbf{3 b} \cdot \mathrm{HCl}, \mathrm{C}_{20} \mathrm{H}_{28} \mathrm{ClNO}_{6}\right)$, methyl 6-[2-(N,N-diethylamino)ethoxy]-7(p-methoxycinnamoyl)-3-methyl-2-benzofurancarboxylate hydrochloride $\left(\mathbf{4 b} \cdot \mathrm{HCl}, \mathrm{C}_{27} \mathrm{H}_{32} \mathrm{ClNO}_{6}\right)$,

Table 4 Intra and intermolecular interactions in crystals of $\mathbf{1 c}\left(\AA{ }^{\circ}\right)$

\begin{tabular}{lllll}
\hline $\mathrm{D}-\mathrm{H} \cdots \mathrm{A}$ & $\mathrm{D}-\mathrm{H}$ & $\mathrm{H} \cdots \mathrm{A}$ & $\mathrm{D} \cdots \mathrm{A}$ & $<(\mathrm{D}-\mathrm{H} \cdots \mathrm{A})$ \\
\hline $\mathrm{O} 17-\mathrm{H} 17 \mathrm{~A} \cdots \mathrm{O} 16$ & 0.82 & 1.70 & $2.433(2)$ & 148 \\
$\mathrm{C} 4-\mathrm{H} 4 \mathrm{~A} \cdots \mathrm{O} 18$ & 0.93 & 2.54 & $3.011(2)$ & 111 \\
$\mathrm{C} 11-\mathrm{H} 11 \mathrm{D} \cdots \mathrm{O} 18$ & 0.97 & 2.28 & $2.637(3)$ & 101 \\
$\mathrm{C} 7-\mathrm{H} 7 \mathrm{~A} \cdots \mathrm{O} 18^{\mathrm{a}}$ & 0.93 & 2.53 & $3.313(2)$ & 179 \\
$\mathrm{C} 11-\mathrm{H} 11 \mathrm{~A} \cdots \mathrm{O} 1^{\mathrm{b}}$ & 0.97 & 2.53 & $3.166(2)$ & 123 \\
$\mathrm{C} 13 \mathrm{D}-\mathrm{H} 13 \mathrm{G} \cdots \mathrm{O} 17^{\mathrm{c}}$ & 0.97 & 2.71 & $3.415(3)$ & 130 \\
$\mathrm{C} 13 \mathrm{C}-\mathrm{H} 13 \mathrm{~F} \cdots \mathrm{O} 16^{\mathrm{d}}$ & 0.97 & 2.67 & $3.435(5)$ & 136 \\
$\mathrm{C} 8-\mathrm{H} 8 \mathrm{~B} \cdots \mathrm{C} 10^{\mathrm{e}}$ & 0.96 & 2.85 & $3.661(3)$ & 143 \\
$\mathrm{C} 15-\mathrm{H} 15 \mathrm{C} \cdots \mathrm{C} 9^{\mathrm{f}}$ & 0.96 & 2.84 & $3.558(3)$ & 133
\end{tabular}

Symmetry codes: ${ }^{\mathrm{a}} 1+\mathrm{x}, \mathrm{y}, \mathrm{z} ;{ }^{\mathrm{b}}-1+\mathrm{x}, \mathrm{y}, \mathrm{z} ;{ }^{\mathrm{c}}-\mathrm{x}, 1-\mathrm{y},-\mathrm{z} ;{ }^{\mathrm{d}}-0.5+\mathrm{x}, 1.5-\mathrm{y}, 0.5+\mathrm{z} ;{ }^{\mathrm{e}} 1-\mathrm{x}, 1-\mathrm{y},-\mathrm{z} ;{ }^{\mathrm{f}} 1-\mathrm{x}, 2-\mathrm{y},-\mathrm{z}$ 
Fig. 4 Projection of the crystal structure of 1c viewed along the $a$ axis, showing molecular blocks

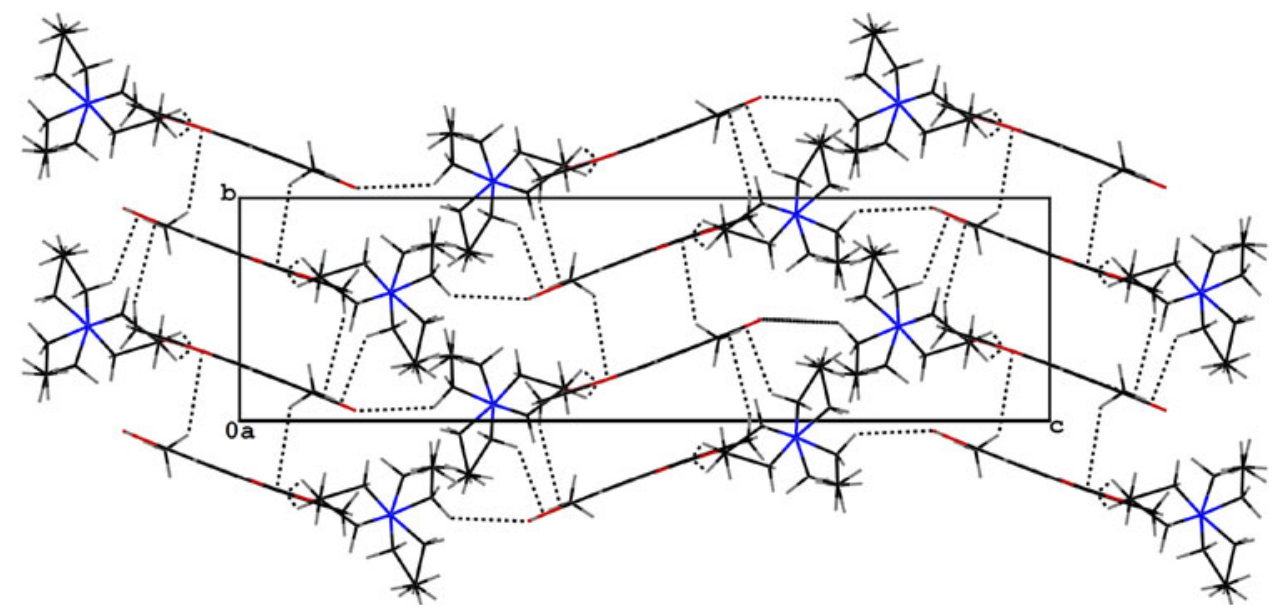

Table 5 Crystal data, data collection, and structure refinement for $\mathbf{1 c}$

\begin{tabular}{|c|c|}
\hline Compound & 1c \\
\hline Empirical formula & $\mathrm{C}_{18} \mathrm{H}_{23} \mathrm{NO}_{5}$ \\
\hline Formula weight & 333.37 \\
\hline$T / \mathrm{K}$ & 293(2) \\
\hline Wavelength/Å & 1.54178 \\
\hline Crystal system, space group & Monoclinic, $P$ 2(1)/n \\
\hline \multicolumn{2}{|l|}{ Unit cell dimensions } \\
\hline$a / \AA$ & $8.1681(1)$ \\
\hline$b / \AA$ & $7.4276(1)$ \\
\hline$c / \AA$ & $27.3017(3)$ \\
\hline$\beta /^{\circ}$ & $94.218(1)$ \\
\hline Volume $/ \AA^{3}$ & $1,651.89(4)$ \\
\hline$Z, D_{x} / \mathrm{mg} \mathrm{m}^{-3}$ & $4,1.340$ \\
\hline$\mu / \mathrm{mm}^{-1}$ & 0.805 \\
\hline$F(000)$ & 712 \\
\hline$\theta$ range for data collection ${ }^{\circ}$ & $5.55-88.24$ \\
\hline$h k l$ range & $\begin{array}{l}-10 \leq h \leq 10 \\
-9 \leq k \leq 7 \\
-33 \leq l \leq 33\end{array}$ \\
\hline \multicolumn{2}{|l|}{ Reflections } \\
\hline Collected & 17,343 \\
\hline Unique $\left(R_{\text {int }}\right)$ & $3,523(0.021)$ \\
\hline Observed $(I>2 \sigma(I))$ & 3,324 \\
\hline Data/restraints/parameters & $3,523 / 0 / 236$ \\
\hline Goodness-of-fit on $F^{2}$ & 1.007 \\
\hline$R(F)[I>2 \sigma(I)]$ & 0.0629 \\
\hline$w R\left(F^{2}\right)$ (all data) & 0.1921 \\
\hline $\operatorname{Max} / \min . \Delta \rho \mathrm{e} / \AA^{-3}$ & $0.323 /-0.278$ \\
\hline
\end{tabular}

methyl 5-bromo-7-[2-(N,N-diethylamino)ethoxy]-6methoxy-2-benzofurancarboxylate hydrochloride $\left(\mathbf{6 b} \cdot \mathrm{HCl}, \mathrm{C}_{17} \mathrm{H}_{22} \mathrm{BrNO}_{5}\right)$

Analytical data $\left({ }^{1} \mathrm{H}\right.$ NMR data and m.p.) for compounds $2 b-4 b$ and $6 \mathbf{b}$ were in agreement with the data reported in our paper [15].
General procedure for microwave-assisted preparation of hydrochlorides of 2-(N,N-diethylamino $)$ ethyl benzofurancarboxylates

The appropriate benzofurancarboxylic acid $(0.3 \mathrm{mmol})$, $\mathrm{N}, \mathrm{N}$-diethyl-2-chloroethylamine hydrochloride $(1.5 \mathrm{mmol})$, anhydrous potassium carbonate $(10.2 \mathrm{mmol})$, and Aliquat $336(0.25 \mathrm{mmol})$ in $8 \mathrm{~cm}^{3}$ anhydrous acetone were placed in the microwave flask. The mixture was heated under reflux in the monomode microwave oven: 4-8 cycles: heating $6 \mathrm{~min}$, cooling $2 \mathrm{~min}$. TLC monitoring on silica gel plates (mobile phase $\mathrm{CHCl}_{3}-\mathrm{MeOH}$ 10:1) indicated complete disappearance of the substrate. The inorganic salts were removed by filtration, then the solvent was evaporated. The residue was purified by column chromatography on silica gel 230-400 mesh, eluent: $\mathrm{CHCl}_{3}-\mathrm{MeOH}$ 50:1. One or two basic products were isolated. The bases were converted into their hydrochlorides as described above.

2-(N,N-Diethylamino)ethyl 6-acetyl-5-hydroxy-2-methyl-3benzofurancarboxylate $\left(\mathbf{1 c}, \mathrm{C}_{18} \mathrm{H}_{23} \mathrm{NO}_{5}\right)$

Yield $61 \%$; m.p.: $101-103{ }^{\circ} \mathrm{C} ; R_{\mathrm{f}}=0.69 ;{ }^{1} \mathrm{H}$ NMR $\left(300 \mathrm{MHz}, \quad \mathrm{CDCl}_{3}\right): \delta=1.10 \quad(\mathrm{t}, \quad 6 \mathrm{H}, \quad J=7.2 \mathrm{~Hz}$, H-14,14'), 2.67 (t, 4H, $\left.J=7.2 \mathrm{~Hz}, \mathrm{H}-13,13^{\prime}\right), 2.68$ (s, $3 \mathrm{H}, \mathrm{H}-15), 2.79$ (s, 3H, H-8), $2.91(\mathrm{t}, 2 \mathrm{H}, J=6.5 \mathrm{~Hz}$, $\mathrm{H}-12), 4.45$ (t, 2H, $J=6.5 \mathrm{~Hz}, \mathrm{H}-11), 7.49$ (s, 1H, H-4), 7.77 (s, 1H, H-7), $12.17(1 \mathrm{H}, \mathrm{OH})$ ppm; ${ }^{13} \mathrm{C}$ NMR $\left(125 \mathrm{MHz}, \mathrm{CDCl}_{3}\right): \delta=11.74\left(\mathrm{C}-14,14^{\prime}\right), 15.13(\mathrm{C}-8)$, 26.94 (C-16), 47.82 (C-13,13'), 51.36 (C-12), 62.28 (C-11), 109.37 (C-3), 109.46 (C-4), 111.97 (C-7), 116.45 (C-6), 134.08 (C-3a), 146.77 (C-7a), 159.45 (C-5), 163.83 (C-9), 169.56 (C-2), 203.88 (C-10) ppm; IR $\left(\mathrm{CHCl}_{3}\right): \bar{v}=3,417$ $\left(v_{\mathrm{OH}}\right), \quad 3,076\left(v_{\mathrm{C}-\mathrm{H}_{\text {arom }}}\right), 2,963,2,926\left(v_{\mathrm{C}-\mathrm{H}_{\text {asym }}}\right), 2,852$ $\left(v_{\mathrm{C}-\mathrm{H}_{\mathrm{sym}}}\right), 1,703\left(v_{\mathrm{C}=\mathrm{O}}\right), 1,621,1,587\left(v_{\mathrm{C}=\mathrm{C}}\right), 1,423\left(\delta_{\mathrm{OH}}\right)$, $1,318,1,260\left(v_{\mathrm{C}-\mathrm{O}}-_{\mathrm{C}_{\text {asym }}}\right), 1,176,1,092\left(v_{\mathrm{C}-\mathrm{O}}-_{\mathrm{C}}{ }_{\text {asym }}\right), 979$, 887, 863, $799\left(\gamma_{\mathrm{C}-\mathrm{H}}\right) \mathrm{cm}^{-1}$; MS (TOF-ES +$):[\mathrm{M}+\mathrm{H}]^{+}$ calcd for $\mathrm{C}_{18} \mathrm{H}_{24} \mathrm{NO}_{5} 334.1654$, found 334.1654. 
1c. $\mathrm{HCl}\left(\mathrm{C}_{18} \mathrm{H}_{24} \mathrm{ClNO}_{5}\right)$

${ }^{1} \mathrm{H}$ NMR $\left(300 \mathrm{MHz}, \mathrm{CDCl}_{3}\right): \delta=1.46(\mathrm{t}, 6 \mathrm{H}, J=7.2 \mathrm{~Hz}$, H-14,14'), 2.79 (s, 3H, H-8), 2.69 (s, 3H, H-16), 3.25 (m, $\left.4 \mathrm{H}, \mathrm{H}-13,13^{\prime}\right), 3.46(\mathrm{~m}, 2 \mathrm{H}, \mathrm{H}-12), 4.94(\mathrm{t}, 2 \mathrm{H}$, $J=5.4 \mathrm{~Hz}, \mathrm{H}-11), 7.35$ (s, 1H, H-4), 7.80 (s, 1H, H-7) ppm, $12.17(1 \mathrm{H}, \mathrm{OH}) \mathrm{ppm} ;{ }^{13} \mathrm{C}$ NMR $\left(125 \mathrm{MHz}, \mathrm{CDCl}_{3}\right)$ : $\delta=8.87\left(\mathrm{C}-14,14^{\prime}\right), 15.41(\mathrm{C}-8), 26.98(\mathrm{C}-16), 47.52(\mathrm{C}-$ 13,13'), 50.13 (C-12), 58.57 (C-11), 108.37 (C-3), 108.90 (C-4), 112.33 (C-7), 116.70 (C-6), 133.43 (C-3a), 146.75 (C-7a), 159.61 (C-5), 163.21 (C-9), 170.51 (C-2), 203.86 (C-10) $\mathrm{ppm}$.

\section{2-(N,N-Diethylamino)ethyl 6-acetyl-5-}

[2-(N,N-diethylamino)ethoxy]-2-methyl-3-

benzofurancarboxylate $\left(\mathbf{1 d}, \mathrm{C}_{24} \mathrm{H}_{36} \mathrm{~N}_{2} \mathrm{O}_{5}\right)$

Yield $71 \%$; m.p.: $101-103{ }^{\circ} \mathrm{C} ; R_{\mathrm{f}}=0.75 ;{ }^{1} \mathrm{H}$ NMR $\left(300 \mathrm{MHz}, \mathrm{CDCl}_{3}\right): \delta=1.068,1.072(\mathrm{t}, 6 \mathrm{H}, J=7.2 \mathrm{~Hz}$; t, $\left.6 \mathrm{H}, J=7.8 \mathrm{~Hz} ; \mathrm{H}-14,14^{\prime}, 14^{\prime \prime}, 14^{\prime \prime \prime}\right), 2.64$ (q, 8H, $\left.J=7.2 \mathrm{~Hz}, \mathrm{H}-13,13^{\prime}, 13^{\prime \prime}, 13^{\prime \prime \prime}\right), 2.70$ (s, 3H, H-15), 2.77 (s, 3H, H-8), 2.87 (t, 2H, $J=6.3 \mathrm{~Hz}, \mathrm{H}-12^{\prime}$ ), 2.95 (t, $2 \mathrm{H}, J=6.3 \mathrm{~Hz}, \mathrm{H}-12), 4.20$ (t, $2 \mathrm{H}, J=6.3 \mathrm{~Hz}, \mathrm{H}-11^{\prime}$ ), 4.44 (t, 2H, $J=6.3 \mathrm{~Hz}, \mathrm{H}-11), 7.54$ (s, 1H, H-4), 7.83 (s,1H, H-7) ppm; MS (TOF-ES+): $[\mathrm{M}+\mathrm{H}]^{+}$calcd for $\mathrm{C}_{24} \mathrm{H}_{37} \mathrm{~N}_{2} \mathrm{O}_{5}$ 433.2702, found 433.2702.

1d. $2 \mathrm{HCl}\left(\mathrm{C}_{24} \mathrm{H}_{38} \mathrm{Cl}_{2} \mathrm{~N}_{2} \mathrm{O}_{5}\right)$

${ }^{1} \mathrm{H} \quad \mathrm{NMR} \quad\left(300 \mathrm{MHz}, \quad \mathrm{CDCl}_{3}\right): \quad \delta=1.44 \quad(\mathrm{t}, \quad 6 \mathrm{H}$, $J=7.2 \mathrm{~Hz}), 1.47\left(\mathrm{t}, 6 \mathrm{H}, J=7.8 \mathrm{~Hz}, \mathrm{H}-14,14^{\prime}\right), 2.63$ (s, $3 \mathrm{H}, \mathrm{H}-16), 2.79$ (s, 3H, H-8), 3.35 (m, 8H, H-13, 13', $\left.13^{\prime \prime}, 13^{\prime \prime \prime}\right), 3.61\left(\mathrm{t}, 2 \mathrm{H}, J=5.1 \mathrm{~Hz}, \mathrm{H}-12^{\prime}\right), 3.72(\mathrm{~m}, 2 \mathrm{H}$, $\mathrm{H}-12), 4.86$ (t, $\left.J=4.8 \mathrm{~Hz}, 2 \mathrm{H}, \mathrm{H}-11^{\prime}\right), 4.98(\mathrm{t}, J=4.8 \mathrm{~Hz}$, 2H, H-11), 7.69 (s, 1H, H-4), 7.76 (s, 1H, H-7), 11.92, 12.04 (br.s, $2 \mathrm{NH})$ ppm; IR $\left(\mathrm{CHCl}_{3}\right): \bar{v}=2,981,2,955$, $2,927\left(v_{\mathrm{C}-\mathrm{H}_{\text {asym }}}\right), 2,855\left(v_{\mathrm{C}-\mathrm{H}_{\text {asym }}}\right), 2,489\left(v_{\mathrm{N}-\mathrm{H}}\right.$ tertiary amine salt $), 1,713\left(v_{\mathrm{C}=\mathrm{O}}\right), 1,623,1,588 \quad\left(v_{\mathrm{C}=\mathrm{C}}\right), 1,440$ $\left(\delta_{\mathrm{C}-\mathrm{H}_{\text {asym }}}\right), \quad 1,250, \quad 1,227 \quad\left(v_{\mathrm{C}-\mathrm{O}-\mathrm{C}_{\text {asym }}}\right), \quad 1,182, \quad 1,090$ $\left(v_{\mathrm{C}-\mathrm{O}-\mathrm{C}_{\mathrm{sym}}}\right), 979,892,847,780\left(\gamma_{\mathrm{C}-\mathrm{H}}\right) \mathrm{cm}^{-1}$.

2-(N,N-Diethylamino)ethyl 7-acetyl-6-hydroxy-5-methoxy3-methyl-2-benzofurancarboxylate $\left(3 \mathbf{c}, \mathrm{C}_{19} \mathrm{H}_{25} \mathrm{NO}_{6}\right)$

Yield $55 \%$; m.p.: $61-63{ }^{\circ} \mathrm{C} ; \quad R_{\mathrm{f}}=0.16 ;{ }^{1} \mathrm{H} \quad \mathrm{NMR}$ $\left(300 \mathrm{MHz}, \quad \mathrm{CDCl}_{3}\right): \delta=1.12 \quad(\mathrm{t}, \quad 6 \mathrm{H}, \quad J=7.2 \mathrm{~Hz}$, $\left.\mathrm{H}-14,14^{\prime}\right), 2.57$ (s, 3H, 8-H), $2.72(\mathrm{q}, 4 \mathrm{H}, J=7.2 \mathrm{~Hz}$, $\left.\mathrm{H}-13,13^{\prime}\right), 2.95$ (s, 3H, H-16), 2.94 (t, $2 \mathrm{H}, J=6.0 \mathrm{~Hz}$, H-12), 3.98 (s, 3H, H-15), 4.93 (t, 2H, $J=6.0 \mathrm{~Hz}, \mathrm{H}-11$ ), 7.15 (s, 1H, H-4), 13.61 (br.s, $1 \mathrm{H}, \mathrm{OH}) \mathrm{ppm} ;{ }^{13} \mathrm{C} \mathrm{NMR}$ $\left(125 \mathrm{MHz}, \mathrm{CDCl}_{3}\right): \delta=9.55(\mathrm{C}-8), 11.72\left(\mathrm{C}-14,14^{\prime}\right)$, 31.76 (C-16), $47.85\left(\mathrm{C}-13,13^{\prime}\right), 51.19$ (C-12), 56.85 (C-15), 62.71 (C-11), 106.93 (C-7), 107.64 (C-4), 119.74 (C-3a), 126.80 (C-3), 140.32 (C-2), 146.97 (C-6), 147.99 (C-5), 156.37 (C-7a), 160.00 (C-9), 203 (C-10) ppm; MS (TOF$\mathrm{ES}+)$ : $[\mathrm{M}+\mathrm{H}]^{+}$calcd for $\mathrm{C}_{19} \mathrm{H}_{26} \mathrm{NO}_{6} 362.1760$, found 364.1760 . 3c. $\mathrm{HCl}\left(\mathrm{C}_{19} \mathrm{H}_{26} \mathrm{ClNO}_{6}\right)$

IR $\left(\mathrm{CHCl}_{3}\right): 3,424\left(v_{\mathrm{OH}}\right), 2,954,2,926\left(v_{\mathrm{C}-\mathrm{H}_{\text {asym }}}\right), 2,855$ $\left(v_{\mathrm{C}-\mathrm{H}_{\text {asym }}}\right), 2,485\left(v_{\mathrm{N}-\mathrm{H}}\right.$ tertiary amine salt $), 1,716\left(v_{\mathrm{C}=\mathrm{O}}\right)$, $1,610, \quad 1,584 \quad\left(v_{\mathrm{C}=\mathrm{C}}\right), \quad 1,428 \quad\left(\delta_{\mathrm{OH}}\right), \quad 1,316, \quad 1,260$ $\left(v_{\mathrm{C}-\mathrm{O}-\mathrm{C}_{\text {asym }}}\right), 1,148,1,097\left(v_{\mathrm{C}-\mathrm{O}-\mathrm{C}_{\text {asym }}}\right), 977,934,848$, 799, $769\left(\gamma_{\mathrm{C}-\mathrm{H}}\right) \mathrm{cm}^{-1}$.

\section{2-(N,N-Diethylamino)ethyl 6-hydroxy-7-}

(p-methoxycinnamoyl)-3-methyl-2-benzofurancarboxylate $\left(4 \mathbf{c}, \mathrm{C}_{26} \mathrm{H}_{29} \mathrm{NO}_{6}\right.$ )

Yield $65 \%$; m.p.: $104-106{ }^{\circ} \mathrm{C} ; R_{\mathrm{f}}=0.59 ;{ }^{1} \mathrm{H}$ NMR $\left(300 \mathrm{MHz}, \quad \mathrm{CDCl}_{3}\right): \quad \delta=1.08 \quad(\mathrm{t}, \quad 6 \mathrm{H}, \quad J=7.2 \mathrm{~Hz}$, $\left.\mathrm{H}-14,14^{\prime}\right), 2.57$ (s, 3H, H-8), 2.67 (m, 4H, H-13,13'), 2.96 (t, 2H, $J=6.5 \mathrm{~Hz}, \mathrm{H}-12), 3.88$ (s, 3H, H-21), 4.53 (t, $2 \mathrm{H}, J=6.5 \mathrm{~Hz}, \mathrm{H}-11), 6.97$ (m, 4H, H-18,18',19,19'), 7.65 $(\mathrm{d}, 1 \mathrm{H}, J=8.7 \mathrm{~Hz}, \mathrm{H}-5), 7.75(\mathrm{~d}, 1 \mathrm{H}, J=8.7 \mathrm{~Hz}, \mathrm{H}-4)$, $7.99(\mathrm{~d}, \quad 1 \mathrm{H}, \quad J=15.3 \mathrm{~Hz}, \quad \mathrm{H}-16), 8.34 \quad(\mathrm{~d}, \quad 1 \mathrm{H}$, $J=15.1 \mathrm{~Hz}, \mathrm{H}-15$ ), 14.06 (br.s, $1 \mathrm{H}, \mathrm{OH})$ ppm; ${ }^{13} \mathrm{C}$ NMR $\left(125 \mathrm{MHz}, \mathrm{CDCl}_{3}\right): \delta=9.40\left(\mathrm{C}-14,14^{\prime}\right), 11.96(\mathrm{C}-$ 8), 29.91 (C-16), 47.81 (C-13,13'), 51.51 (C-12), 55.69 (C21), 62.86 (C-11), 107.35 (C-3), 114.79 (C-19,19'), 116.03 (C-5), 121.64 (C-7), 122.69 (C-4), 127.92 (C-17), 128.35 (C-3a), 131.11 (C-18, 18'), 140.19 (C-7a), 145.94 (C-15), 153.59 (C-20), 160.01 (C-6), 162.36 (C-9), 166.54 (C-2), 191.85 (C-10) ppm; IR $\left(\mathrm{CHCl}_{3}\right): \bar{v}=3,400\left(v_{\mathrm{OH}}\right), 2,965$, $2,925\left(v_{\mathrm{C}-\mathrm{H}_{\text {asym }}}\right), 2,851\left(v_{\mathrm{C}-\mathrm{H}_{\text {asym }}}\right), 1,712\left(v_{\mathrm{C}=\mathrm{O}}\right), 1,635$, $1,593\left(v_{\mathrm{C}=\mathrm{C}}\right), 1,424\left(\delta_{\mathrm{OH}}\right), 1,259\left(v_{\mathrm{C}-\mathrm{O}-\mathrm{C}_{\text {asym }}}\right), 1,172,1,085$ $\left(v_{\mathrm{C}-\mathrm{O}-\mathrm{C}_{\text {asym }}}\right), 983,869,829,764\left(\gamma_{\mathrm{C}-\mathrm{H}}\right) \mathrm{cm}^{-1}$; MS (TOF$\mathrm{ES}+):[\mathrm{M}+\mathrm{H}]^{+}$calcd for $\mathrm{C}_{26} \mathrm{H}_{30} \mathrm{NO}_{6} 452.3222$, found 452.3222 .

\section{2-(N,N-Diethylamino)ethyl 5-bromo-6,7-dimethoxy-2-}

benzofurancarboxylate $\left(\mathbf{6 c}, \mathrm{C}_{17} \mathrm{H}_{22} \mathrm{BrNO}_{5}\right)$

This compound was prepared in accordance with the general procedure, except acetone-methanol 9:10 was used instead of anhydrous acetone. Yield $48 \%$; m.p.: $61-63{ }^{\circ} \mathrm{C}$; $R_{\mathrm{f}}=0.52 ;{ }^{1} \mathrm{H}$ NMR $\left(300 \mathrm{MHz}, \mathrm{CDCl}_{3}\right): \delta=1.11(\mathrm{t}, 6 \mathrm{H}$, $\left.J=7.2 \mathrm{~Hz}, \mathrm{H}-14,14^{\prime}\right), 2.75$ (q, 4H, $J=7.2 \mathrm{~Hz}, \mathrm{H}-13,13^{\prime}$ ), 3.00 (t, 2H, $J=6.3 \mathrm{~Hz}, \mathrm{H}-12), 3.92$ (s, 3H, H-10), 3.95 (s, $3 \mathrm{H}, \mathrm{H}-9), 4.44(\mathrm{t}, 2 \mathrm{H}, J=6.3 \mathrm{~Hz}, \mathrm{H}-11), 7.12(\mathrm{~s}, 1 \mathrm{H}$, 3-H), 7.46 (s, 1H, 4-H) ppm; MS (TOF-ES+): $[\mathrm{M}+\mathrm{H}]^{+}$ calcd for $\mathrm{C}_{17} \mathrm{H}_{23} \mathrm{NO}_{5} \mathrm{Br}^{79}$ 400.0753, found 400.1958; $\mathrm{C}_{17} \mathrm{H}_{23} \mathrm{NO}_{5} \mathrm{Br}^{81}$ 402.0733, found 402.2709.

6c. $\mathrm{HCl}\left(\mathrm{C}_{17} \mathrm{H}_{23} \mathrm{BrClNO}_{5}\right)$

${ }^{1} \mathrm{H}$ NMR $\left(300 \mathrm{MHz}, \mathrm{CDCl}_{3}\right): \delta=1.14(\mathrm{t}, 6 \mathrm{H}, J=7.5 \mathrm{~Hz}$, $\left.\mathrm{H}-14,14^{\prime}\right), 2.82$ (m, 2H, H-13,13'), 3.05 (m, 2H, H-12), 3.92 (s, 3H, H-10), 3.95 (s, 3H, H-9), 4.62 (t, 2H, $J=6.3 \mathrm{~Hz}, \mathrm{H}-11), 7.13(\mathrm{~s}, 1 \mathrm{H}, 3-\mathrm{H}), 7.46(\mathrm{~s}, 1 \mathrm{H}, 4-\mathrm{H})$ ppm; IR $\left(\mathrm{CHCl}_{3}\right): \bar{v}=2,967,2,932\left(v_{\mathrm{C}-\mathrm{H}_{\text {asym }}}\right), 2,848$ $\left(v_{\mathrm{C}-\mathrm{H}_{\text {asym }}}\right), \quad 1,731 \quad\left(v_{\mathrm{C}=\mathrm{O}}\right), \quad 1,619,1,581,1,502 \quad\left(v_{\mathrm{C}=\mathrm{C}}\right)$, $1,262\left(v_{\mathrm{C}-\mathrm{O}-\mathrm{C}_{\text {asym }}}\right), 1,122\left(v_{\mathrm{C}-\mathrm{O}-\mathrm{C}_{\text {asym }}}\right), 982,914,834$, $764\left(\gamma_{\mathrm{C}-\mathrm{H}}\right) \mathrm{cm}^{-1}$. 
2-(N,N-Diethylamino)ethyl 7-methoxy-2-

benzofurancarboxylate $\left(\mathbf{7 c}, \mathrm{C}_{16} \mathrm{H}_{21} \mathrm{NO}_{4}\right)$

Yield $55 \%$; oil; $R_{\mathrm{f}}=0.86 ;{ }^{1} \mathrm{H}$ NMR $\left(300 \mathrm{MHz}, \mathrm{CDCl}_{3}\right)$ : $\delta=1.09\left(\mathrm{t}, 6 \mathrm{H}, J=7.2 \mathrm{~Hz}, \mathrm{H}-14,14^{\prime}\right), 2.66(\mathrm{q}, 4 \mathrm{H}$, $\left.J=6.9 \mathrm{~Hz}, \mathrm{H}-13,13^{\prime}\right), 2.89(\mathrm{t}, 2 \mathrm{H}, J=6.3 \mathrm{~Hz}, \mathrm{H}-12)$, 4.02 (s, 3H, H-10), 4.58 (t, 2H, $J=6.3 \mathrm{~Hz}, \mathrm{H}-11), 6.92$ (dd, 1H, $J=7.2 \mathrm{~Hz}, 1.5 \mathrm{~Hz}, \mathrm{H}-5), 7.24$ (m, 2H, H-4, H-6), 7.51 (s, 1H, H-3) ppm; MS (TOF-ES+): $[\mathrm{M}+\mathrm{H}]^{+}$calcd for $\mathrm{C}_{16} \mathrm{H}_{22} \mathrm{NO}_{4} 292.1555$, found 292.1549.

\section{7c. $\mathrm{HCl}\left(\mathrm{C}_{16} \mathrm{H}_{22} \mathrm{ClNO}_{4}\right)$}

IR $\left(\mathrm{CHCl}_{3}\right): \bar{v}=2,979,2,950\left(v_{\mathrm{C}-\mathrm{H}_{\text {asym }}}\right), 2,843\left(v_{\mathrm{C}-\mathrm{H}_{\text {asym }}}\right)$, $2,485\left(v_{\mathrm{N}_{-\mathrm{H}}}\right.$ tertiary amine salt $), 1,727\left(v_{\mathrm{C}=\mathrm{O}}\right), 1,622$, $1,594\left(v_{\mathrm{C}=\mathrm{C}}\right), 1,366,1,307,1,271\left(v_{\mathrm{C}-\mathrm{O}-\mathrm{C}_{\text {asym }}}\right), 1,185$, $1,093\left(v_{\mathrm{C}-\mathrm{O}} \mathrm{C}_{\mathrm{asym}}\right), 972,914,850,800,780,732\left(\gamma_{\mathrm{C}-\mathrm{H}}\right)$ $\mathrm{cm}^{-1}$.

\section{Microbiology}

The following microbial strains with different cell wall structures were chosen:

- Gram-positive bacteria: Micrococcus luteus ATCC 9341, B. cereus ATCC 11778, B. subtilis ATCC 6633, S. epidermidis ATCC 12228, S. aureus ATCC 6538, S. aureus ATCC 6538P, E. hirae ATCC 10541;

- Gram-negative bacteria: E. coli ATCC 8739, P. aeruginosa ATCC 15442; and

- fungal strains: Aspergillus brasiliensis ATCC 16404, C. albicans ATCC 10231 and ATCC 2091, $C$. parapsilosis ATCC 22019, S. cervisiae ATCC 9763, Z. rouxi ATCC 28253 .

The cylinder-plate method was used in the preliminary antimicrobial activity tests [18]. A suspension of the tested compound $\left(20 \mathrm{mg} / \mathrm{cm}^{3}, 0.05 \mathrm{~cm}^{3}\right.$, in $0.08 \mathrm{M}$ phosphate buffer, pH 7.0, containing $10 \%$ DMSO) was placed in the cylinder. The cylinders were placed on a Muller-Hinton 2 or Sabouraud agar plate inoculated with one of the tested strains. The bacterial strains were incubated at $37{ }^{\circ} \mathrm{C}$ for $24 \mathrm{~h}$ and the fungal strains at $30^{\circ} \mathrm{C}$ for $48 \mathrm{~h}$. Minimal inhibitory concentration (MIC) was obtained by mixing with $19 \mathrm{~cm}^{3}$ Mueller-Hinton 2 agar and cooling to $56^{\circ} \mathrm{C}$ with $1 \mathrm{~cm}^{3}$ of the appropriate dilution of the tested compound. Then, $2 \times 10^{-3} \mathrm{~cm}^{3}$ of a particular cell suspension of optical density 0.5 unit on the McFarland scale was applied to the surface of the agar. The lowest concentration of tested compound which totally inhibited growth of the examined strain was evaluated as MIC value [19]. For control samples, MIC values of ciprofloxacin ranged between 0.14 and $0.37 \times 10^{-3} \mu \mathrm{mol} / \mathrm{cm}^{3}$ for bacterial strains and MIC values of fluconazole ranged between $3.9 \times 10^{-4}$ and $8.4 \times 10^{-1} \mu \mathrm{mol} / \mathrm{cm}^{3}$ for yeast strains.
Crystallography

Crystals of 1c suitable for X-ray analysis were grown by slow evaporation of a solution in toluene-isopropanol (1:1). Diffraction data were collected on an Oxford Diffraction SuperNova diffractometer using $\mathrm{CuK}_{\alpha}$ radiation at room temperature. Data reduction was performed with SuperNova software [20]. The unit cell parameters were determined by least-squares treatment of setting angles of the highest-intensity reflections chosen from the whole experiment. The structure was solved by direct methods, by use of SHELXS-97 software, and refined on $F^{2}$ by the fullmatrix least-squares method, again by use of SHELXL97 software [21]. Two reflections were excluded from the reflection file because of their large $\left(\left|F_{2}\right|^{2}-\left|F_{c}\right|^{2}\right)$ differences. The function $\Sigma w\left(\left|F_{\mathrm{o}}\right|^{2}-\left|F_{\mathrm{c}}\right|^{2}\right)^{2}$ was minimized with $w^{-1}=\left[\sigma^{2}\left(F_{\mathrm{o}}\right)^{2}+(0.1234 P)^{2}+0.3568 P\right]$, where $P=$ $\left(F_{\mathrm{o}}^{2}+2 F_{\mathrm{c}}^{2}\right) / 3$.

Non-hydrogen atoms were refined with anisotropic thermal data and the atoms of $O$-aminoethyl substituent were found to be disordered. So, the C12, C13A, and C13B atoms were located in two alternative positions and their occupancies were refined to $0.487(5)$ for $\mathrm{C} 12 \mathrm{~A} / \mathrm{C} 13 \mathrm{~A} / \mathrm{C} 13 \mathrm{~B}$ and $0.513(5)$ for $\mathrm{C} 12 \mathrm{~B} / \mathrm{C} 13 \mathrm{C} / \mathrm{C} 13 \mathrm{D}$. The coordinates of the hydrogen atoms were generated geometrically and refined "riding" on their parent atoms with $U_{\text {iso }}$ set at 1.2 (1.5 for methyl group) times $U_{\text {eq }}$ of the appropriate carrier atom. All details concerning data collection, crystal data, and structure refinement are given in Table 5. The supplementary information in the CIF form is available from Cambridge Crystallographic Database Centre, no. CCDC-949328.

Acknowledgments The project was supported by the Medical University of Warsaw, Faculty of Pharmacy, Grant FW 24/PM1/11.

Open Access This article is distributed under the terms of the Creative Commons Attribution License which permits any use, distribution, and reproduction in any medium, provided the original author(s) and the source are credited.

\section{References}

1. De Luca L, Nieddu G, Porcheddu A, Giacomelli G (2009) Curr Med Chem 16:1

2. Engelmeier D, Hadacek F, Pacher T, Vajrodaya S, Greger H (2000) J Agric Food Chem 48:1400

3. Jiang X, Liu W, Zhang W, Jiang F, Gao Z, Zhuang H, Fu L (2011) Eur J Med Chem 46:3526

4. Georgopapadakou NH (2002) Expert Opin Investig Drugs 8:1117

5. Masubuchi M, Kawasaki K, Ebiike H, Ikeda Y, Tsujii S, Sogabe S, Fujii T, Sakata K, Shiratori Y, Aoki Y, Ohtsuka T, Shimma N (2001) Bioorg Med Chem Lett 11:1833

6. Ebiike H, Masubuchi M, Liu P, Kawasaki K, Morikami K, Sogabe S, Hayase M, Fujii T, Sakata K, Shindoh H, Shiratori Y, 
Aoki Y, Ohtsuka T, Shimma N (2002) Bioorg Med Chem Lett 12:607

7. Masubuchi M, Ebiike H, Kawasaki K, Sogabe S, Morikami K, Shiratori Y, Tsujii S, Fujii T, Sakata K, Hayase M, Shindoh MH, Aoki Y, Ohtsuka T, Shimma N (2003) Bioorg Med Chem $11: 4463$

8. Kawasaki K, Masubuchi M, Morikami K, Sogabe S, Aoyama T, Ebiike H, Niizuma S, Hayase M, Fujii T, Sakata K, Shindoh H, Shiratori Y, Aoki Y, Ohtsuka T, Shimma N (2003) Bioorg Med Chem Lett 13:87

9. Khan NW, Alam MJ, Rashid MA, Chowdhury R (2005) Bioorg Med Chem 13:4796

10. Abdel-Aziz HA, Mekawey AAI, Dawood KM (2009) Eur J Med Chem 44:3637

11. Logoglu E, Yilmaz M, Katircioglu H, Yakut M, Mercan S (2010) Med Chem Res 19:490

12. Kossakowski J, Krawiecka M, Kuran B, Stefańska J, Wolska I (2010) Molecules 15:4737

13. Krawiecka M, Kuran B, Kossakowski J, Wolska I, Kierzkowska M, Młynarczyk G (2012) Acta Polon Pharm Drug Res 69:1055

14. Trykowska Konc J, Hejchman E, Kruszewska H, Wolska I, Maciejewska D (2011) Eur J Med Chem 46:2252

15. Kossakowski J, Ostrowska K, Hejchman E, Wolska I (2005) Farmaco 60:519

16. Macrae CF, Edgington PR, McCabe P, Pidcock E, Shields GP, Taylor R, Towler M, van de Streek J (2006) J Appl Cryst 39:453
17. Frisch MJ, Trucks GW, Schlegel HB, Scuseria GE, Robb MA, Cheeseman JR, Scalani G, Barone V, Mennucci B, Petersson GA, Nakatsuji H, Caricato M, Li X, Hratchian HP, Izmaylov AF, Oblino J, Zheng G, Sonnenberg JL, Hada M, Ehara M, Toyota K, Fukusa R, Hasegawa J, Ishida M, Nakajima T, Honda Y, Kitao O, Nakai H, Vreven T, Montgomery JA, Peralta JE, Ogliaro F, Bearpark M, Hyde JJ, Brothers E, Kunin KN, Staroverov VN, Keith T, Kobayashi R, Normand J, Raghavachari K, Rendell A, Burant JC, Iyengar SS, Tomasi J, Cossi M, Rega N, Millam JM, Klene M, Knox JE, Cross JB, Bakken V, Adamo C, Jaramillo J, Gomperts R, Stratmann RE, Yazyev O, Austin AJ, Cammi R, Pomelli C, Ochterski JW, Martin RL, Morokuma K, Zakrzewski VG, Voth GA, Salvador P, Dannenberg JJ, Dapprich S, Daniels AD, Farkas O, Foresman JB, Ortiz JV, Cioslowski J, Fox DJ (2010) Gaussian 09, Revision B.01. Gaussian, Inc, Wallingford, CT

18. European Pharmacopoeia (2008) In: The European Directorate for the Quality of Medicines, Council Of Europe, 6th edn. Strasbourg, France

19. National Committee for Clinical Laboratory Standards (2003) Approved standard M7-A6, 6th edn. NCCLS, Wayne

20. Agilent Technologies (2011) CrysAlisPRO, version 1.171.35.15

21. Sheldrick GM (2008) Acta Crystallogr A64:112 